\title{
EPIDERMOLYSIS BULLOSA - A REPORT OF TWO CASES
}

H. Murat Akgül, O. Murat Bilge, A. Berhan Yilmaz. Epidermolysis bullosa - a report of two cases. Annal Dent Univ Malaya 2002; 9: 27-33.

\section{SUMMARY}

Epidermolysis bullosa is a group of rare dermal diseases characterized by hereditary and nonhereditary vesicular disorders of skin and mucous membranes that result from trauma or heat.

In this report, two patients suffering from epidermolysis bullosa simplex and junctional epidermolysis bullosa were described.

Key words: Epidermolysis bullosa, junctional, simplex, regional odontodysplasia.

\section{INTRODUCTION}

Epidermolysis Bullosa (EB) is a group of rare dermal diseases producing blister spontaneously or after minor trauma, characterized by vesicular disorders of skin and mucous membranes (1-9). The disease may have autosomal dominant and recessive inheritance or may be acquired thereafter $(1,3,9,10)$. The most distinctive clinical feature of the disease is the formation of vesicles and bullae as a result of minor mechanical traumas. The lesions generally occur on the skin but they also commonly appear on the mucosa. Over twenty subtypes of $\mathrm{EB}$ have been defined by the genetical and clinical studies that have been carried out so far $(10,11)$. The types of the disease are defined based on the ultrastructural level of tissue cleavage followed by mechanical traumas (dermolytic, epidermolytic or lamina lucidolytic), clinical characteristics (scarring or non-scarring), and mode of inheritance (autosomal dominant or autosomal recessive) $(1,5,9,12-16)$.

The most generally acknowledged and at the same time the simplest classification is the one based on the anatomical level of tissue cleavage (1).

- Type I. Intraepidermal forms (Epidermolysis bullosa simplex).

- Type II. Junctional forms (Junctional epidermolysis bullosa).

- Type III. Dermal forms (Epidermolysis bullosa dystrophica dominant and recessive).

- Type IV. Epidermolysis bullosa acquisita.

The differential diagnosis should include Kindler syndrome, bullous impetigo (pemphigus neonatorum), Ritter's disease, porphyria congenita, congenital
Case Report

H. Murat Akgül ${ }^{1}$, O. Murat Bilge ${ }^{2}$ and

A. Berhan Yilmaz

${ }^{I}$ Assistant Professor

${ }^{2}$ Professor

${ }^{3}$ Associate Professor

Department of Oral Diagnosis and Oral Radiology Faculty of Dentistry, Atatürk University, Erzurum, 25240 Turkey

Business Tel: 0090.442.2311766

Home Tel: 0090.442.2353950

Fax: 0090.442.2360945

Email:makgul@atauni.edu.ti

Corresponding author-Dr. H. Murat Akgül

syphilis, juvenile bullous dermatitis herpetiformis, pemphigus, bullous pemphigoid, bullous erythema multiforme, linear IgA disease, or drug eruptions $(1,10,12,17)$.

The purpose of this study is to present 2 patients diagnosed as epidermolysis bullosa simplex and junctional epidermolysis bullosa according to the literature.

\section{CASE 1}

An eight-year-old boy presented with complaints of toothache. He was found to have extensive tooth decay. In the general history of the disease there were no indication of the presence of epidermolysis bullosa. A more detailed history showed a history of abrasions on the heel just after birth. It was also reported that oral bullae developed because of the irritation of the tissue during chewing, which healed shortly after.

A general examination showed the patient presenting with distinctive nail malformations (Fig. 1) and demonstrated evidence of abraded skin during infancy. An intraoral examination showed extensive tooth decay due to dental neglect and poor mouth care and enamel hypoplasia (Fig. 2). The tongue seemed to be normal but movement was restricted by the presence of oral bullae.

The radiographic examination showed no loss of the primary or permanent teeth but the upper left first premolar tooth had an appearance suggestive of regional odontodysplasia (Fig. 3).

Under the light of these findings, supported by the history of the disease and clinical examination, the initial diagnosis was epidermolysis bullosa simplex (EBS). This diagnosis was confirmed upon consultation 


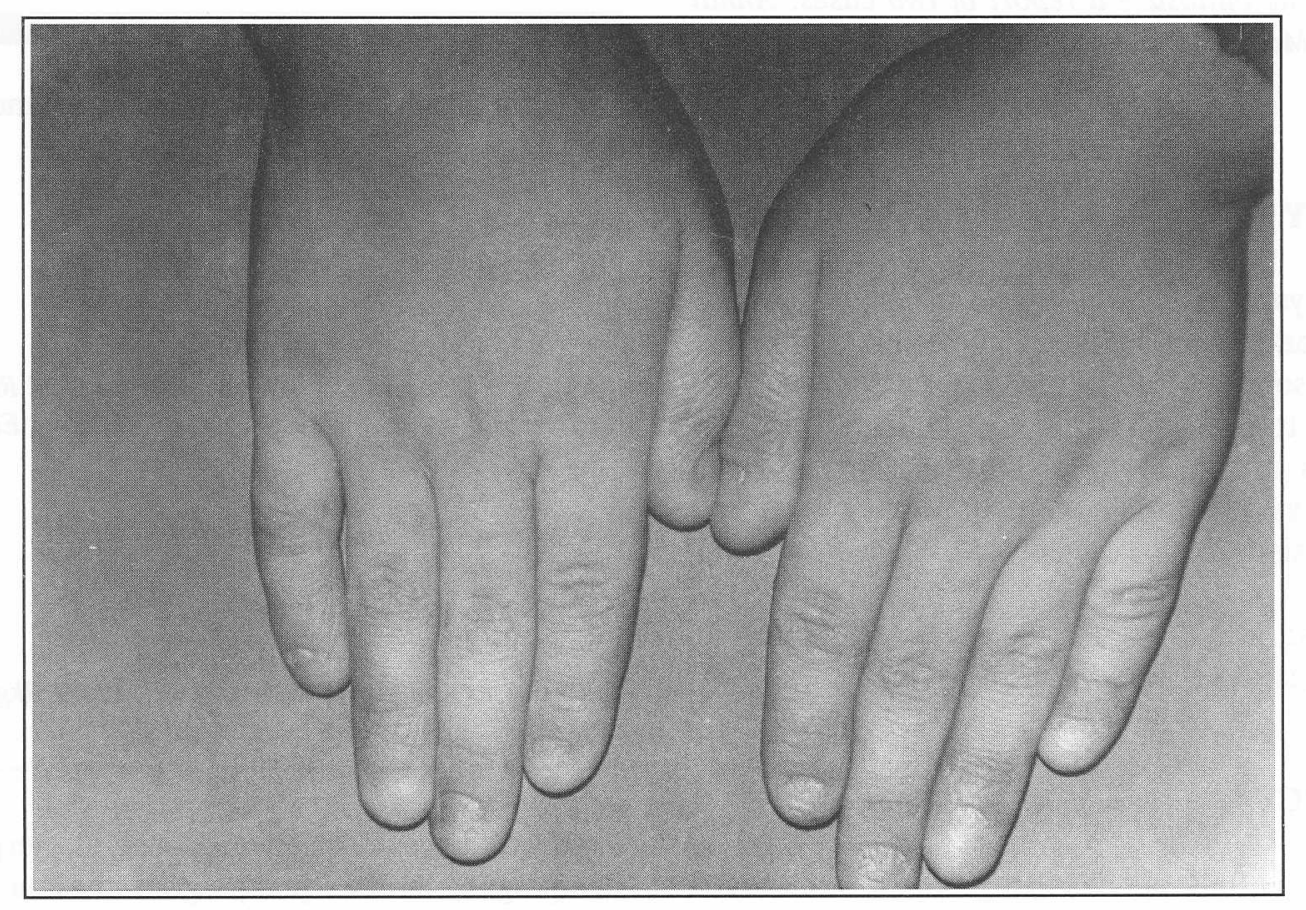

Figure 1: Dorsal view of hands, showing nails malformations in patient with EBS.

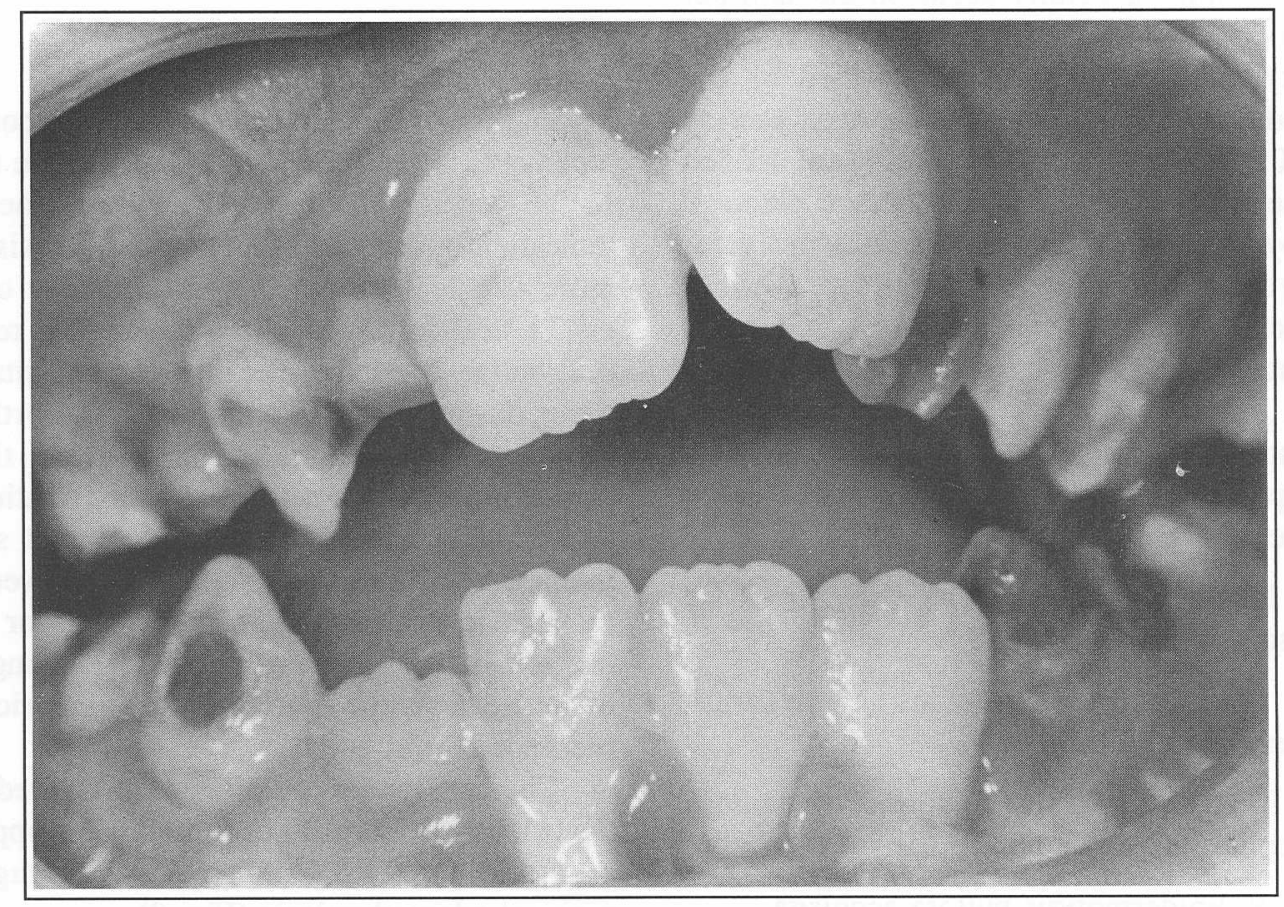

Figure 2: Extensive tooth decay and enamel hypoplasia in patient with EBS. 


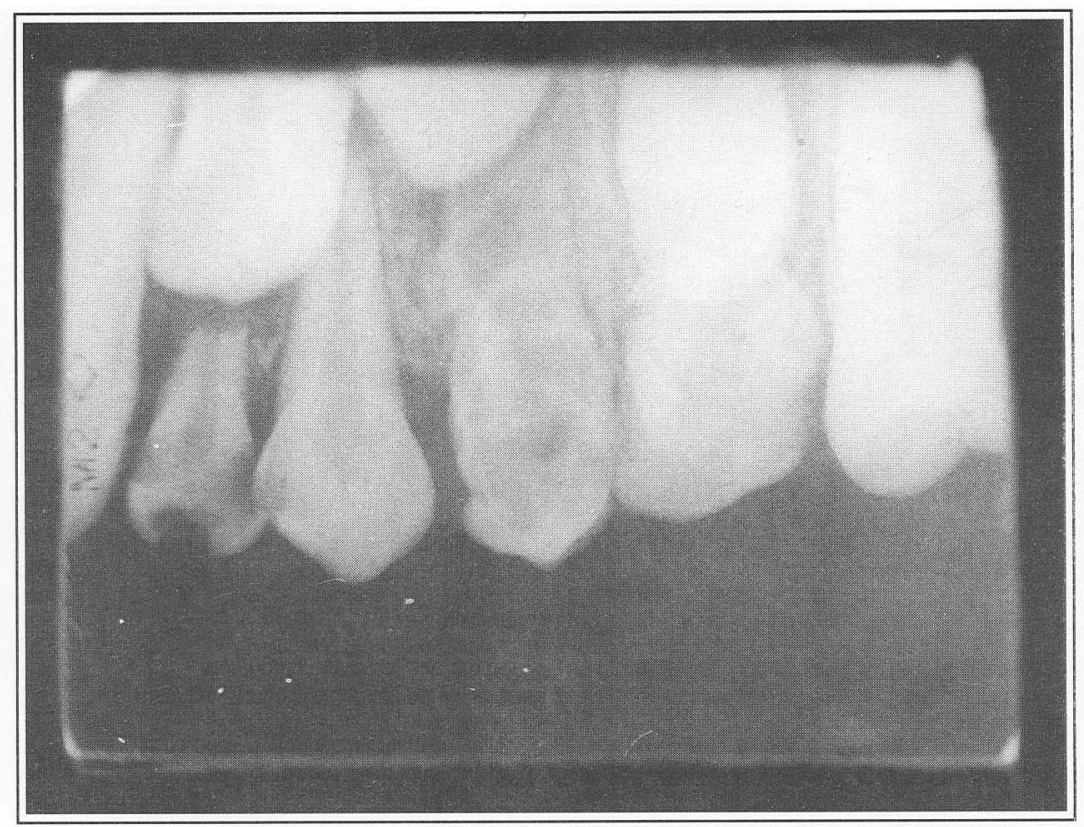

Figure 3: Upper left first premolar tooth suggesting regional odontodysplasia in patient with EBS.

with the Department of Dermatology, Faculty of Medicine, Atatürk University.

Atraumatic treatments of the carious teeth were performed. The parents of the patient were adviced to clean the child's teeth and gingiva with a piece of cottonwool or sponge and to wash his mouth out twice a day with gargles containing chlorhexidine.

\section{CASE 2}

This is a seven-year-old girl with complaints of persistent toothache. The history of her disease showed that blisters developed on her face, arms and legs below the knees. The skin became abraded afterwards. When she was one and a half or two years of age, the nails of her hands and feet became loose and shed. A diagnosis of junctional epidermolysis bullosa (JEB) was reached based on the history, clinical findings, a histopathological report of a biopsy performed at the Department of Dermatology of the Ege University. The biopsy reported the development of subepidermal bullae, accumulation of fibrinogen at the site of separation observed by immunoflorescent method, and the presence of basal membrane at the base of the bullae when stained with periodic acid Schiff reagent.

The extraoral examination of the patient showed atrophic nails (Fig. 4), sparsely abraded skin of the face and particularly of joints, and the development of integumental skin (Fig. 5). There was also split changes of pigmentation and milia.

The intraoral examination demonstrated extensive ulceration on buccal and labial mucosa, palates and on the dorsal and lateral surfaces of the tongue. The tongue surface was smooth and glossy due to loss of lingual papillae (Fig. 6). The painful ulcers prevented effective oral hygiene measures. The gingiva was hypertrophic and shiny in appearance. There was extensive calculus, tooth caries and enamel defects. The anterior incisors seemed to be dystrophic. Chewing and gulping of food was difficult because of extensive erosions inside the mouth due to formation of vesicles and bullae with increased traumas.

Radiographic investigation revealed that the permanent tooth germs were normal, there was no missing tooth and chronic apical abscesses were evident associated with both the lower right and left first premolar teeth. The abscess associated teeth were extracted atraumatically 3 days later with the administration of antibiotics and lubrication of the tips and edges of the forceps to avoid irritation. Oral toilet was advocated using cotton-wool or spongue and chlorhexidine mouthwash.

\section{DISCUSSION}

EB is a dermal disorder resulting in a number of clinical features such as delayed wound healing, risk of secondary infection, malnutrition, dental neglect and poor mouth care and an unpleasant non-aesthetic appearance (18). The severity and frequency of oral and dermal lesions is in accordance with the type of disorder.

Epidermolysis bullosa simplex is a type of $\mathrm{EB}$ that is inherited as an autosomal dominant trait $(1,3,4,12,16)$. Histopathologically, as a result of acute degeneration of basal cells of the epidermis, a cleavage at the level of the basal cell layer was observed which is associated with the development of intraepidermal 


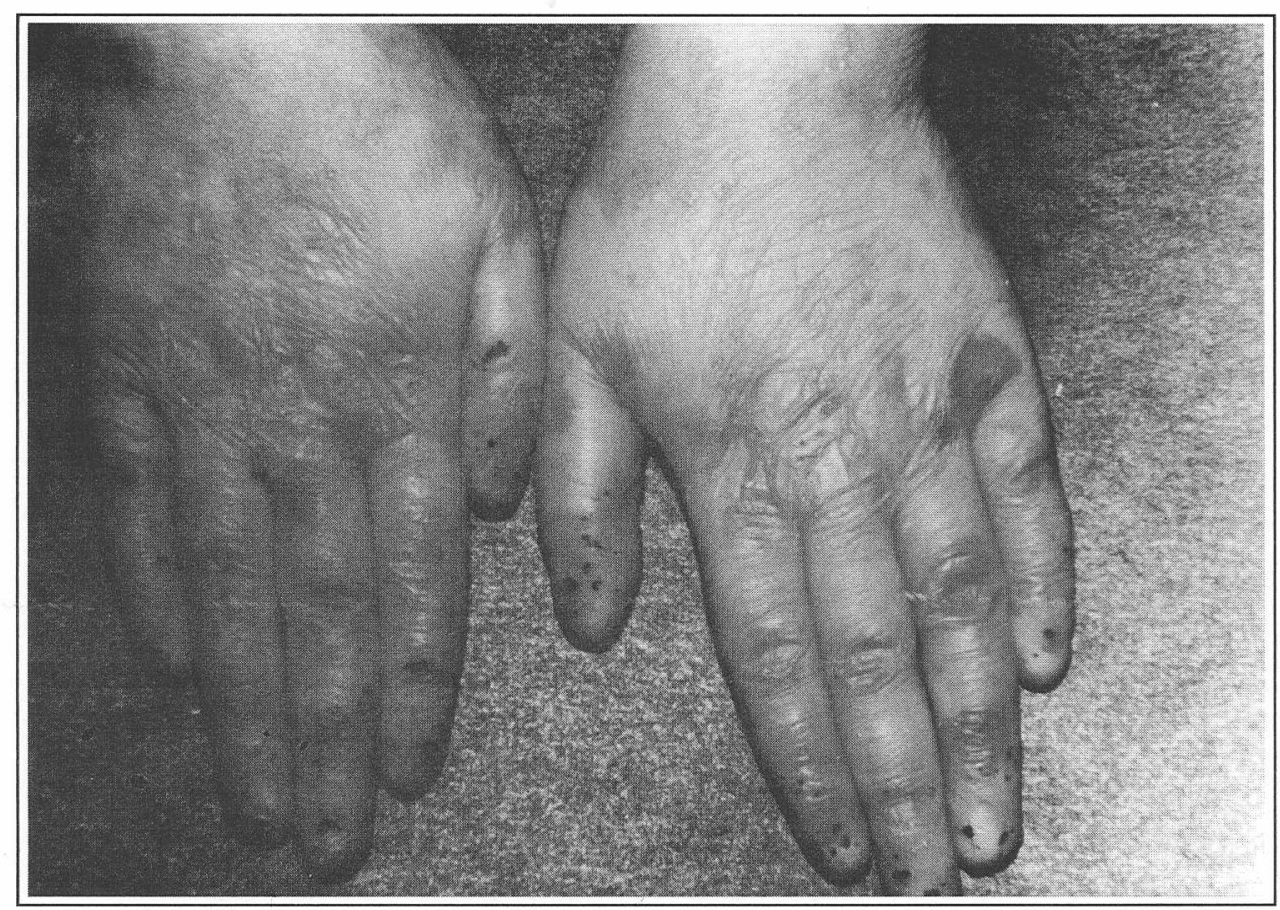

Figure 4: Atrophic nails and changes of pigmentation in patient with JEB.

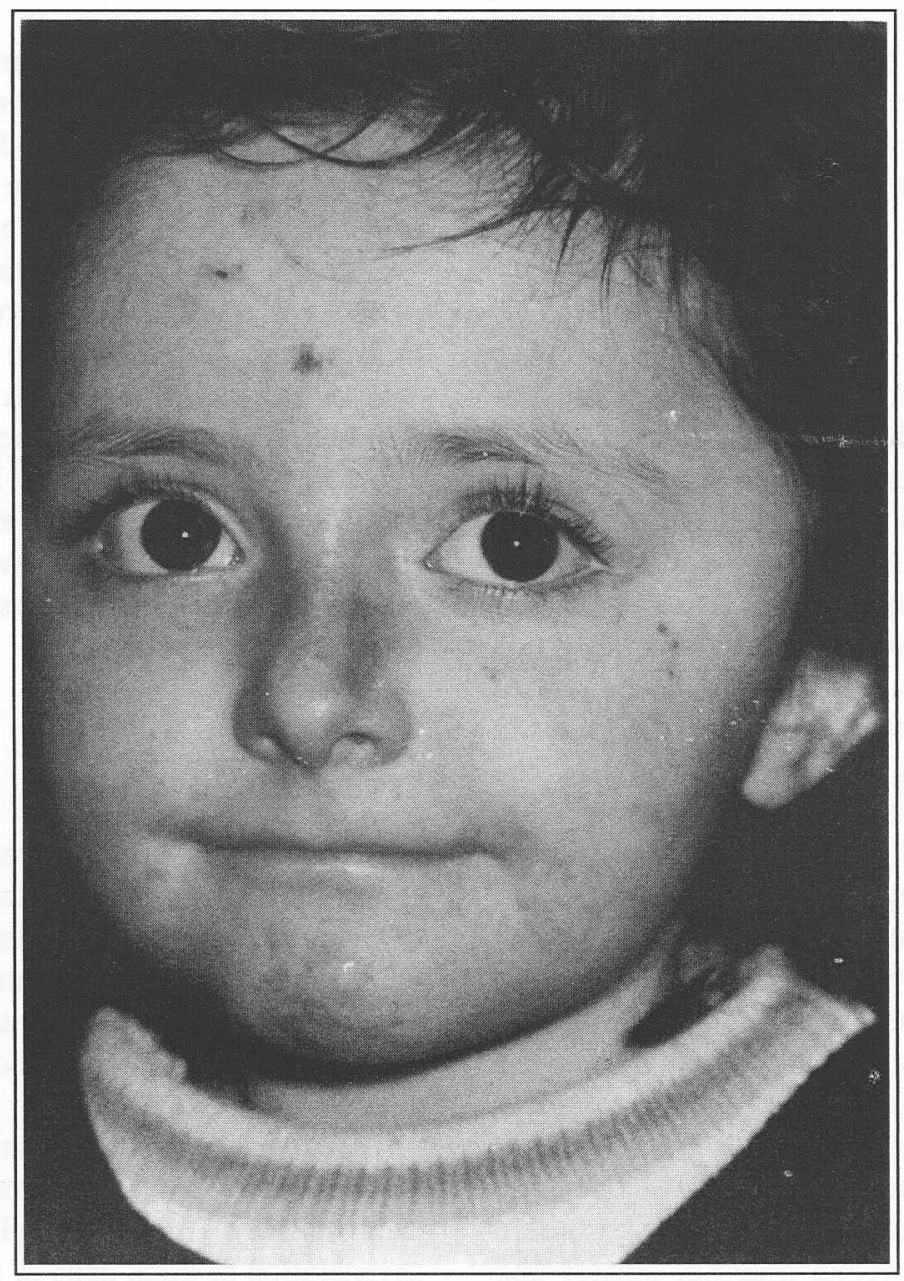

Figure 5: Abraded and integumental skin of the face in patient with JEB. 


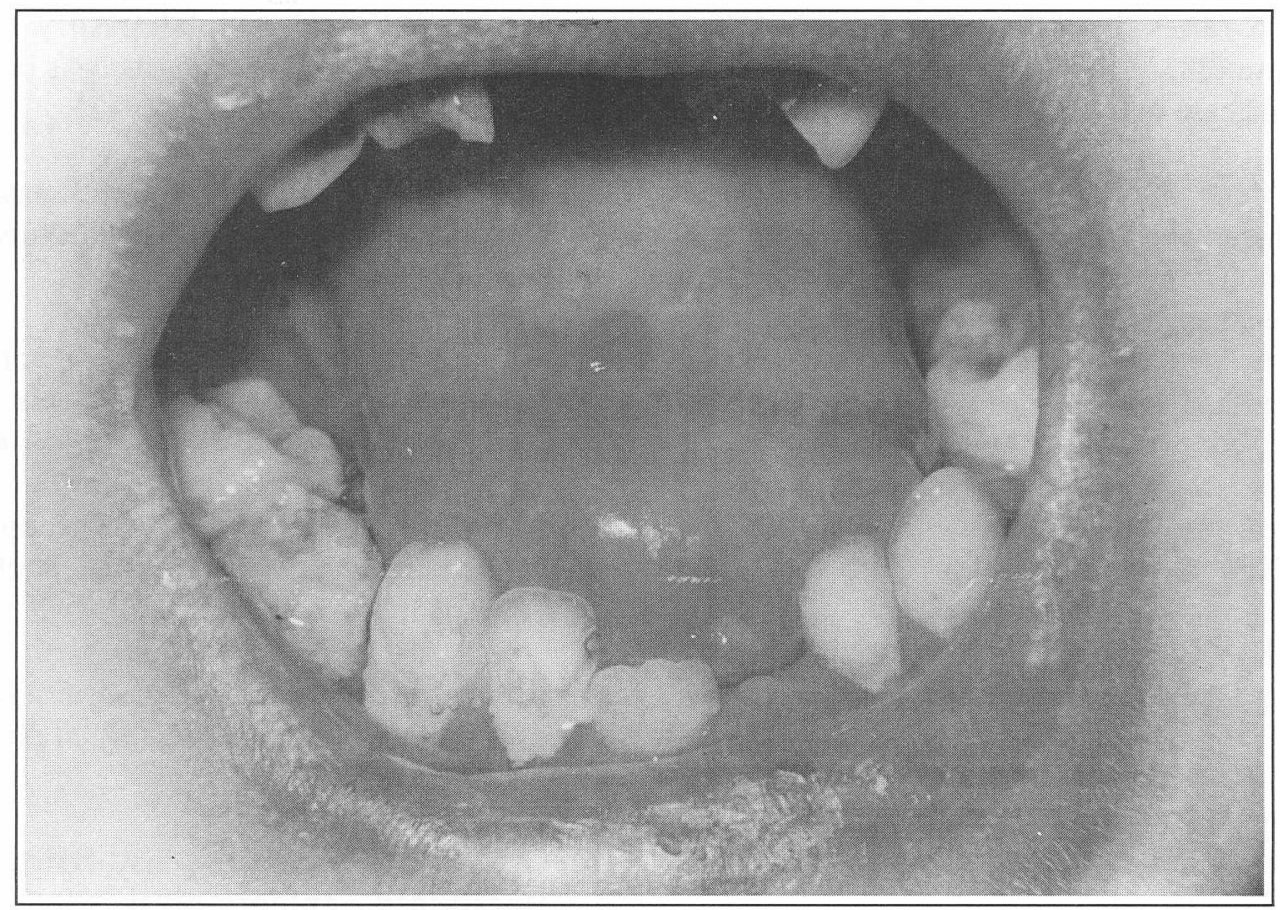

Figure 6: Smooth and glossy tongue surface, and enamel defects in patient with JEB.

bullae $(11,16,17)$. The disease generally appears at or shortly after birth $(3,7,12)$. It principally involves the hands and the feet, and rarely the ankles, the knees, the trunk and the elbows $(1,4)$. The frequency of bullae development may be precipitated by an increase in room temperature and lesions heal without leaving any scarring or pigmentation (1-4,12). Nails and extracutaneous organs are rarely affected (3). In 20 percent of cases nail malformations are reportedly seen $(1,4)$. The frequency and distribution of enamel defects are nearly similar to those of normal individuals $(2,5,19)$. Contrary to previous beliefs $(4,16)$, oral lesions may be observed in this subtype of epidermolysis bullosa. Wright et al. (13), report that milia and oral lesions were seen in 21 per cent and 40 per cent of the cases, respectively.

In the first case of this report, diagnosed as EBS, the occurrence of skin lesions and nail malformations was in accordance with the types of diseases defined in the literature eventhough no milia were observed. However, the current reported case also showed oral lesions which were extensive enough to restrict the movement of the tongue, while widespread enamel hypoplasia and regional odontodysplasia in the upper left first premolar tooth were also evident. Such a case has not been previously reported in patients with EB.

This type of EB has been reported as having quite a good prognosis and heals spontaneously at puberty (3).

Junctional epidermolysis bullosa (JEB) is a rare genetic disease with autosomal recessive transmission. Histological investigation under the electron microscope revealed a line cleavage between the plasma membrane of the basal cells and the basal membrane, and within the lamina lucida of the dermoepidermal junction $(6,9,11,20,21)$.

It was first described by Herlitz in $1935(6,22)$. Herlitz Syndrome is characterized clinically by widespread blistering with erosions of the skin and the mucous membranes (23). This type of EB was initially called epidermolysis bullosa hereditaria letalis because a number of fatal cases were experienced but this term was discarded later when some infant patients survived into adulthood and thus it was replaced by the term junctional epidermolysis bullosa. JEB has two major sub-groups called Herlitz and Non-Herlitz. In the former the symptoms are more serious and some patients die of sepsis in the early months of life while the mortality rate is comparatively lower in the latter. Other clinical features are similar to those of the former $(1,6,23,24)$.

Blisters are produced at birth or develop shortly thereafter because of minor mechanical trauma $(6,7,12)$. In the early months of life, hemorrhagic vesicles arise at the base of the fingernails $(4,25)$. Almost in all patients fragile and hemorrhagic bullae develop at the junction of soft and hard palates (26). Dysphagia was reported to arise in the 15 per cent of patients $(4,8)$. Although scars, intraoral and extraoral milia have reportedly been seen, these findings are not frequently observed in JEB $(13,17)$.

Teeth may be rudimentary or congenitally absent and there is a high rate of caries activity $(5,27,28)$. In all JEB cases enamel defects were reported. Generalized enamel hypoplasia is peculiar to both types of JEB though it is more seriously in Herlitz's (5). All of the teeth or a limited number may be affected by these factors. Patients with recessive 
dystrophic and junctional epidermolysis bullosa are more prone to caries formation than those in other types of disease (28).

The second case in this report, presenting a patient believed to suffer from JEB, had nail malformations, skin lesions along with sparse pigmentation changes and milia. There were also dysphagia, mucosal bullae and ulceration. These findings including the presence of extensive enamel hypoplasia and tooth caries were in conformity with the findings reported in the literature, eventhough there was no absence of teeth observed.

\section{REFERENCES}

1. Sedano HO, Gordin RJ. Epidermolysis bullosa. Oral Surg Oral Med Oral Pathol 1989; 67: 55563.

2. Hochberg MS, Vazquez-Santiago IA, Sher M. Epidermolysis bullosa. Oral Surg Oral Med Oral Pathol 1993; 75: 54-7.

3. Moghadam BK, Gier RE. Epidermolysis bullosa: oral management and case reports. ASDC J Dent Child 1992; 59: 66-9.

4. Gorlin RJ. Epidermolysis bullosa. Oral Surg Oral Med Oral Pathol 1971; 32: 761-6.

5. Wright JT, Johnson LB, Fine JD. Developmental defects of enamel in humans with hereditary epidermolysis bullosa. Arch Oral Biol 1993; 38: 945-55.

6. Gardner DG, Hudson CD. The disturbances in odontogenesis in epidermolysis bullosa hereditaria letalis. Oral Surg Oral Med Oral Pathol 1975; 40: 483-93.

7. Regezi JA, Sciubba JJ. Oral pathology: clinical pathologic correlations. 3rd edn. Philadelphia W.B. Saunders Company 1999; 25-7.

8. Travis SPL, McGrath JA, Turnbull AJ, Schofield OM, Chan O, O'Connor AF et al. Oral and gastrointestinal manifestations of epidermolysis bullosa. Lancet 1992; 340: 1505-6.

9. Pearson RW. Clinicopathologic types of epidermolysis bullosa and their nondermatological complications. Arch Dermatol 1988; 124: 718-25.

10. Olsen CB, Bourke LF. Recessive dystrophic epidermolysis bullosa. Two case reports with 20 year follow-up. Aust Dent J 1997; 42: 1-7.
11. Fine JD, Bauer EA, Briggaman RA, et al. Revised clinical and laboratory criteria for subtypes of inherited epidermolysis bullosa. J Am Acad Dermatol 1991; 24: 119-35.

12. Laskaris G. Color atlas of oral disease. 2 nd edn. New York Thieme Medical Publishers 1994; 24 6.

13. Wright JT, Fine JD, Johnson LB. Oral soft tissues in hereditary epidermolysis bullosa. Oral Surg Oral Med Oral Pathol 1991; 71: 440-6.

14. Richter BJ, McNutt NS. The spectrum of epidermolysis bullosa acquisita. Arch Dermatol 1979; 115: 1325-8

15. Block MS, Gros BD. Epidermolysis bullosa dystrophica recessive. J Oral Maxillofac Surg 1982; 40: 753-8.

16. Crawford EG, Burkes EJ, Briggaman RA. Hereditary epidermolysis bullosa: Oral manifestations and dental therapy. Oral Surg Oral Med Oral Pathol 1976; 42: 490-500.

17. Elder D, Elenitsas R, Jaworsky C, Johnson B. Histopathology of the skin. 8th edn. Philadelphia Lippincott-Raven Publishers 1997; 128-49.

18. Carter DM, Lin AN. Would healing and epidermolysis bullosa. Arch Dermatol 1988; 124 : 732-3.

19. Haber RM, Ramsay CA, Boxall LBH. Epidermolysis bullosa simplex with keratoderma of the palms and soles. J Am Acad Dermatol 1985; 12: 1040-4.

20. Pearson RW, Potter B, Strauss F. Epidermolysis bullosa hereditaria letalis. Arch Dermatol 1974; 109: 349-55.

21. Wright JT, Childers NK, Evans KL, Johnson LB, Fine JD. Salivary function of persons with hereditary epidermolysis bullosa. Oral Surg Oral Med Oral Pathol 1991; 71: 553-9.

22. Schachner L, Lazarus GS, Dembitzer H. Epidermolysis bullosa hereditaria letalis. Br J Dermatol 1977; 96: 51-8.

23. Verrando $\mathrm{P}$, Blanchet-Bardon $\mathrm{C}$, Pisani A, Thomas JU, Cambazard F, Eady RAJ et al. Monoclonal antibody GB3 defines a widespread defect of several basement membranes and a keratinocyte dysfunction in patients with lethal junctional epidermolysis bullosa. Lab Invest 1991; 64: 85-92. 
24. Tidman MJ, Eady RA. Hemidesmosome heterogeneity in junctional epidermolysis bullosa revealed by morphometric analysis. J Invest Dermatol 1986; 86: 51-6.

25. Haber RM, Hanna W, Ramsay CA, Boxall LB. Cicatricial junctional epidermolysis bullosa. J Am Acad Dermatol 1985; 12: 836-44.

26. Arwill T, Bergenholtz A, Thilander $\mathrm{H}$. Epidermolysis bullosa hereditaria. The ultrastructure of oral mucosa and skin in four cases of the letalis form. Acta Pathol Microbial Scand 1968; 74: 311-24.
27. Arwill T, Bergenholtz A, Olsson O. Epidermolysis bullosa hereditaria; a histologic study of changes in teeth of the polydysplastic dystrophic anhd lethal forms. Oral Surg 1965; 19: 724-44.

28. Wright JT, Capps, J, Fine JD, Johnson LB. Dental caries variation in the different epidermolysis bullosa diseases.J Dent Res 1989; 68: 416. 